\title{
CUESTIONES CRÍTICAS EN LOS CRITERIOS DE SELECCIÓN DE TRABAJADORES AFECTADOS POR LOS DESPIDOS COLECTIVOS EN EL SECTOR PÚBLICO
}

\author{
Critical Issues Related to the Selection Criteria \\ in the Designation of the Workers Affected By Collective Dismissals \\ in Public Sector
}

María Isabel Ribes Moreno

Profesora Ayudante Doctora de Derecho del Trabajo y de la Seguridad Social,

Universidad de Cádiz

\section{ABSTRACT}

La introducción de los despidos colectivos en el Sector público ha suscitado importantes controversias, respecto a la aplicación de los criterios de selección en la designación de los concretos trabajadores afectados por el despido. Algunas de estas controversias parten de la existencia, o no, de diferencias en la aplicación de estas reglas según se trate de una extinción dentro del sector público administrativo, o en el sector público empresarial. En este escenario se destacan dos cuestiones: en primer lugar, la aplicación de la prioridad de permanencia de los trabajadores fijos sobre los temporales y, en segundo lugar, y más importante, el respeto, al establecer los referidos criterios, a los principios de igualdad, mérito y capacidad en la determinación de los trabajadores, proscribiendo toda arbitrariedad. En el presente trabajo se abordarán ambas cuestiones, analizando la regulación y la interpretación judicial, y proponien- 
do la necesidad de introducir reglas claras y adecuadas para evitar la problemática que puede suscitarse en la aplicación de los mencionados criterios.

Palabras clave: despido colectivo, sector público, administración pública, criterios de selección, prioridad de permanencia.

The introduction of a new regulation of collective dismissal in public administration originates controversies, specially related to the selection criteria in the designation of the workers affected by the dismissal. Some controversies arise from the differences in the implementation of rules depending on the nature of the public Administration affected by the measure. This paper analyse two aspects related to this situation: firstly, the application of the priority of permanence between the temporary and regular workers and, secondly, more important, the respect to the merit, equality and capacity principles in the selection of workers, without arbitrariness. The pages that follow analyse such regulation and its judicial interpretation, and propose new adequate and proper rules to avoid the problems deriving from this situation.

Keywords: collective dismissal, public sector, public administration, selection criteria, priority of permanence. 


\section{SUMARIO}

Sumario: 1. Planteamiento previo. 2. Diferencias entre el procedimiento para la designación de las personas afectadas por los despidos colectivos en el Sector público y la empresa privada: un sistema dual. 3. Dos puntos críticos respecto a los despidos colectivos en el Sector Público: la prioridad de permanencia y los criterios de selección. 3.1 La prioridad de permanencia en despidos colectivos. 3.2. La introducción de criterios de selección. 4. Los criterios de selección y la prioridad de permanencia, un análisis de las decisiones judiciales frente a las extinciones en las Administraciones públicas. 5. Reflexiones finales. 6. Bibliografía.

\section{Planteamiento previo}

Las Administraciones públicas, al objeto de conseguir una mayor agilidad y adaptación en su organización y funcionamiento, han ido integrando empleados en régimen laboral dentro de su estructura interna. Tanto es así, que ha sido necesaria la construcción de la acepción de empleado público, recogida dentro de las normas reguladoras del personal al servicio de la administración, y que engloba no solo a los funcionarios públicos, sino también, a los empleados en régimen laboral ${ }^{1}$. Igualmente, siguiendo este mismo objetivo, las normas vigentes han permitido la implantación, en determinadas actividades o servicios, de la llamada "Administración paralela», es decir, la formada por entes, organismos y entidades que se rigen fundamentalmente por el Derecho privado - aunque con excepciones-, materializando en lo que respecta al régimen jurídico de estas entidades una huida del Derecho público en favor del Derecho privado. Todo ello conforma una superestructura que integra el sector público, que constituye un concepto amplio, que integra tanto el sector administrativo, o jurídico público, como el empresarial y fundacional (De Soto, 2015: 86). Estos fenómenos han permitido que, en lo que respecta a las relaciones laborales, hayan de apli-

1 En este sentido, el Real Decreto Legislativo 5/2015, de 30 de octubre, por el que se aprueba el texto refundido de la Ley del Estatuto Básico del Empleado Público (BOE de 31 de octubre), en adelante EBEP, en su artículo 8 establece que son empleados públicos quienes desempeñan funciones retribuidas en las Administraciones Públicas al servicio de los intereses generales diferenciando cuatro categorías dentro de ellos: los funcionarios de carrera, funcionarios interinos, el personal laboral, ya sea fijo, por tiempo indefinido o temporal y el personal eventual. 
carse al sector público las normas del Derecho del Trabajo, pero que requieren de adaptaciones en su regulación, ya que manifiestan dificultades en su encaje ${ }^{2}$.

Entre estas disposiciones sobresalen, por su complejidad, las referentes a los despidos colectivos, cuya regulación general, contenida en el artículo 51 del Estatuto de los Trabajadores, se complementa con las especialidades propias para el sector público que, se introdujeron mediante la Ley 3/2012, de 6 de julio, de medidas urgentes para la reforma del mercado laboral (BOE de 7 de julio), y fueron desarrolladas por el Real Decreto 1483/2012, de 29 de octubre (BOE de 30 de octubre), (en adelante RDDC) ${ }^{3}$. La Ley 3/2012 vino a incluir una Disposición Adicional Vigésima al derogado Estatuto de los Trabajadores, Real Decreto Legislativo 1/1995, de 24 de marzo, (BOE de 29 de marzo), hoy situada, pero reproduciendo los mismos términos, en la Disposición Adicional Decimosexta del vigente, Real Decreto Legislativo 2/2015, de 23 de octubre, por el que se aprueba el texto refundido de la Ley del Estatuto de los Trabajadores (BOE de 24 de octubre). Dicha norma resolvió el debate existente sobre la aplicación de los artículos 51 y 52 del Estatuto de los Trabajadores al sector público, aunque limitándolos al personal laboral a su servicio (Rodríguez-Piñero, 2013: 224). Por tanto, la regulación del despido colectivo para el sector público se recoge conjuntamente con la regulación del despido colectivo para el sector privado, si bien, ajustando el procedimiento con disposiciones específicas a la medida de sus particularidades (García, 2013), según se verá.

La aplicación de la institución del despido colectivo se dirige a poder hacer efectivas medidas de "regulación de empleo»" ${ }^{4}$ aunque limitado al personal laboral al servicio de los entes, organismos y entidades que forman parte del sector público, de acuerdo con lo previsto en el artículo 3.1 del Real Decreto Legislativo 3/2011, que engloba tanto a la Administración General, Autonómica y Local, como a distintos entes, organismos y entidades.

El régimen jurídico que se prevé para la aplicación de los despidos colectivos al ámbito de las Administraciones públicas, parte de «la existencia de una bifurcación de regímenes (De Soto, 2015: 100). Por una parte, cuando se aplique a la Administración pública propiamente dicha y, por otra parte, las demás ma-

2 Estos mecanismos ha pretendido equiparar los objetivos del sector privado con los del sector público, cuestión nada fácil, puesto que el sector privado va dirigido al logro de un beneficio económico y, en cambio, el público se centra "en la defensa de los intereses generales que el ordenamiento juridico le asigna y que, en la mayoría de las supuestos, se concretan en prestaciones de servicios a los ciudadanos que suponen gastos sin generar ingresos», tal como lo expresa García (2013: 2).

${ }^{3} \mathrm{Si}$ bien han sido numerosas las reglas que se han introducido, sobre este particular, en un período muy breve de tiempo. Puede consultarse un breve apunte sobre esta cuestión en De Soto (2015: 98-99).

${ }^{4}$ Término que si bien no tiene razón de ser utilizado para el sector privado, en lo que respecta al público puede mantenerse habida cuenta de sus particularidades, en ese sentido, puede apreciarse el papel que tiene el informe vinculante que figura en el art. 47 del RD 1483/2012. 
nifestaciones del sector público, que la ley denomina «entes, organismos o entidades» y que comprenden a los sujetos que se constituyen en virtud de lo previsto por las normas de Derecho privado (sociedades y fundaciones), o las de Derecho público. Asimismo, cabe realizar una nueva segmentación de regímenes, dependiendo del tamaño, cuando se trate de despidos colectivos en el ámbito de las Administraciones públicas, conforme a lo previsto en los artículos 34 y siguientes del RD 1483/2012 (De Soto, 2015: 101). Es decir, a efectos procedimentales se distinguen, en virtud de la ley, dos situaciones distintas, por un lado, la situación del personal laboral al servicio de los entes, organismos y entidades que no tengan la consideración de Administración Pública, que comparten con las empresas privadas la regulación ordinaria, y por otro lado, la de los restantes supuestos que forman parte del sector público y que cuentan con una regulación ad hoc.

Ahora bien, como se ha dicho, este régimen jurídico diferenciado requiere especificar quién constituye el sector público empresarial —que, como se ha dicho, comparte con el sector privado la regulación del procedimiento- y el sector público administrativo, cuestión que adolece de enorme complejidad (López, 2012: 1-5). Esta clasificación distintiva se realiza conforme establece el Texto Refundido de la Ley de Contratos del Sector Público, RD Legislativo $3 / 2011$, de 14 de noviembre (BOE de 16 de noviembre) $)^{5}$, que dispone en su artículo 3.2 que el sector público administrativo se compone en primer lugar, por la propia Administración pública, que comprende en sentido estricto tanto a la Administración General del Estado (incluidas las Diputaciones), Administración Autonómica y Administración Local; en segundo lugar, por las Entidades Gestoras y Servicios Comunes de la Seguridad Social; en tercer lugar, organismos autónomos; en cuarto lugar, las universidades públicas; en quinto lugar, las entidades de derecho público que tengan atribuidas funciones de regulación o control de carácter externo sobre un determinado sector o actividad, con independencia funcional o con una especial autonomía reconocida por la Ley, y/o vinculadas a una o varias Administraciones Públicas o dependientes de ellas, que no tengan carácter empresarial ni comercial ${ }^{6} \mathrm{y}$, por último, las Diputaciones Forales y las Juntas Generales de los Territorios Históricos del País Vasco. Así, entenderá como tales aquellas entidades que se nutren de recursos públicos

5 Y estableciendo, a tal efecto, un encuadre de lo que se habrá de entender por sector público, si bien la legislación administrativa ofrece distintas definiciones de Administración, lo origina una maraña de situaciones que plantean problemas diversos frente a la estructura organizativa, que resulta difícil de determinar en numerosas ocasiones. Sobre estas cuestiones pueden consultarse numerosas fuentes doctrinales (De Soto, 2015: 10-111 y Purcalla, 2015: 3-6).

${ }^{6}$ A tal efecto, se entenderán como tales aquellas cuya actividad principal no sea la elaboración de productos y servicios destinados al consumo, ni efectuar operaciones de redistribución de la renta y riqueza nacional (no teniendo ánimo de lucro), y/o que no se financien mayoritariamente con ingresos obtenidos de operaciones de entrega de bienes o prestación de servicios. 
(Purcalla, 2015: 3-6). En lo que respecta al sector público empresarial, según la misma norma, éste se conforma por las entidades empresariales públicas ligadas a la Administración o entidades de derecho público que realizan actividades mercantiles, sociedades mercantiles con participación pública mayoritaria y el sector público fundacional ${ }^{7}$.

No obstante, la incorporación de esta medida al sector público manifiesta ciertos puntos de debate que han sido destacados por la doctrina científica (Rodríguez-Pińero, 2013: 219-253; Cantero, 2012: 7-39). Entre las cuestiones que se han suscitado subrayo, por su especial importancia, los criterios de selección de los trabajadores afectados que, a mi entender, han de vincularse a la elección de los más idóneos para el desempeño de la actividad. Ahora bien, esta decisión ha de justificarse perfectamente, puesto que la Administración en su actuación debe ser ajena a toda arbitrariedad, cumpliendo en todo caso con el principio de igualdad. Sin embargo, no existen actualmente criterios o mecanismos legalmente establecidos que pudieran dirigir su conducta en esta dirección, más que los que se concretan respecto a la prioridad de permanencia.

Por lo tanto, en el presente trabajo estableceré en primer lugar, los procedimientos para la realización de los despidos colectivos en el Sector público distinguiendo, dentro de dicho sector, las posibilidades existentes para los colectivos que engloban dicho sector. En segundo lugar, analizaré los dos problemas que se manifiestan al concretar e identificar a los trabajadores afectados, por un lado, las prioridades de permanencia previstas en la norma, especialmente las referidas al personal laboral fijo, ante la extinción colectiva en el sector público y, por otro lado, valorando lo (no) previsto en la norma para la elaboración de los criterios de selección de los sujetos a la medida extintiva. A continuación, abordaré, en concreto, la problemática que afecta a la Administración pública "pura» respecto a los criterios de selección de trabajadores, con revisión de la jurisprudencia que ha tratado este tema. Por último, ofreceré propuestas de modificación que permiten, a mi juicio, una adecuación de la actuación de la Administración a criterios ajenos a cualquier arbitrariedad.

\section{Diferencias entre el procedimiento para la designación de las personas afectadas por los despidos colectivos en el Sector público y la empresa privada: un sistema dual}

Como se ha referido anteriormente, el despido colectivo debe atender en su procedimiento a lo previsto en el RDDC. Así, el Título III de dicha norma in-

\footnotetext{
7 Con una sistemática exposición de la clasificación realizada por el RD Legislativo 3/2011, de 14 de noviembre (Roqueta, 2015: 84-85).
} 
troduce disposiciones específicas aplicables en los despidos colectivos del personal laboral al servicio de los entes, organismos y entidades que forman parte del sector público, si bien, será de aplicación el procedimiento general previsto en el Título I para todo lo que no figure recogido en el Título III, siempre que no se oponga, contradiga o sea incompatible con él ${ }^{8}$. Por tanto, siguiendo lo previsto para los despidos colectivos en general, habrá de establecerse, necesariamente, en la comunicación de inicio del período de consultas, cualquiera que sea la causa alegada, el número y clasificación profesional de los trabajadores afectados, así como, los criterios para la designación de los mismos?

El RDDC prevé, aunque solo para las Administraciones públicas, que estas menciones deberán ir acompañadas de información sobre los criterios tenidos en cuenta en relación con el establecimiento de la prioridad de permanencia del personal laboral fijo que hubiera adquirido esta condición conforme a los principios de igualdad, mérito y capacidad a través de un procedimiento selectivo de ingreso ${ }^{10}$. En cualquier caso, los representantes de los trabajadores deben tener información suficiente que les permita valorar la aplicación de los criterios al trabajador en concreto, criterios que pueden ser matizados, o ampliados, durante la realización del período de consultas ${ }^{11}$.

Ahora bien, sobre esta cuestión se manifiesta una separación entre el empleador público y el privado. Así, los empleadores privados pueden actuar con discrecionalidad a la hora de seleccionar los puestos que van a amortizar mediante el procedimiento de despido colectivo, habida cuenta de la inexistencia de criterios concretos, a diferencia de lo que sucede en otros ordenamientos jurídicos (Ginès, 2010: 1-11). Sin embargo, otra cosa sucederá cuando quien actúe como empleador, y por tanto seleccione a los trabajadores afectados por la extinción, sea una Administración pública, ya que está sometida en su funcionamiento y actuación a mandatos constitucionales ${ }^{12}$. Por tanto, debe actuar con-

8 Tal como se prevé en el art. 34.4 RD 1483/2012.

${ }^{9}$ Cuestión que se introduce en el artículo 3.1 del RD 1483/2012. Esta norma modificó la regulación sobre este particular prevista en el reglamento precedente, RD 803/2011, de 10 de junio, por el que se aprueba el Reglamento de los procedimientos de regulación de empleo y de actuación administrativa en materia de traslados colectivos (BOE de 14 de junio), hoy derogado, en cuyo artículo 8 se establecía que la solicitud que el empresario debía presentar ante la Autoridad laboral, documentando el expediente de regulación de empleo, incorporaba, pero con carácter alternativo, bien una relación nominativa de los trabajadores afectados, o bien, los criterios concretos que hubieran sido tenidos en cuenta para dicha afectación.

10 Por otra parte, en lo que respecta a la prioridad de permanencia, el referido RD 1483/2012 incluye dicha exigencia en el artículo 38.2.

${ }^{11}$ En este sentido se ha pronunciado, en distintas ocasiones, el Tribunal Supremo, aunque con respecto a despidos colectivos en el sector privado, realizando una interpretación anti formalista de la norma de aplicación, vid. STS 17 de julio 2014 (recurso de Casación núm. 32/2014).

12 Entre los que figura el artículo 9 de la CE en el que se establece la interdicción de arbitrariedad de los poderes públicos, y en este sentido se manifiesta Rodríguez-Piñero (2013: 250). 
forme a criterios verdaderamente objetivos "como corresponde coherentemente a una Administración» (De Sande, 2006: 432).

La determinación de un criterio preferente en lo que respecta a los despidos colectivos en la empresa privada han sido interpretados en numerosas ocasiones por la jurisprudencia, habiendo sido considerado como una manifestación del poder de dirección y organización que corresponde al empresario aunque sometido a los límites generales de los derechos fundamentales y libertades públicas, dentro del que se encuentra la prohibición de un trato discriminatorio ${ }^{13}$. En estos supuestos, la decisión debe fundamentarse en criterios de razonabilidad — con ausencia de fraude de ley y abuso de derecho-, y coherentemente con los fines buscados, sin que pueda designarse a los concretos trabajadores de forma caprichosa o arbitraria ${ }^{14}$, aunque con un margen de discrecionalidad. Asimismo, en esta actuación habrán de respetar, como es visible, aquellas normas que pudiesen limitar su libertad como las reglas legales de prioridad de permanencia.

Los límites anteriormente señalados concurren, y se intensifican necesariamente, cuando el empleador pertenece al sector público; más aún cuando se trata de una Administración pública, cuya actuación debe ir orientada a servir con objetividad los intereses generales, en consecuencia, garantizando la selección de los trabajadores más adecuados para cumplir dicho interés ante la posible extinción de contratos ${ }^{15}$. Sin embargo, aunque la Administración pública debe someterse a reglas precisas para garantizar, en su actividad como empleador, el derecho de todos los ciudadanos al acceso al empleo público y, a tal efecto, habrá de actuar conforme a los principios constitucionales de igualdad, mérito y capacidad; en lo que respecta a la extinción no parece que la ley establezca que tenga que utilizarse este mismo criterio, o por lo menos no de forma exclusiva, cuando designe a los trabajadores concretos ${ }^{16}$. Ello es así, puesto que la normativa laboral vigente no recoge criterios específicos a destinados ordenar la actuación de la Administración al seleccionar a los trabajadores que pudieran verse afectados por el despido, tan solo ofrece reglas orientativas referidas a las prioridades de permanencia, así como, recomendaciones de carácter general.

13 Entre otras, STS de 19 de enero de 1998 (recurso de casación para la unificación de doctrina núm. 1460/1997) y de 15 de octubre de 2003 (recurso de casación para la unificación de doctrina núm. 1205/2003).

14 TSJ de Madrid (Sala de lo Social, Sección 3.a) de 30 diciembre de 2013, (recurso de Suplicación núm. 1978/2013).

15 El artículo $103 \mathrm{CE}$ establece que la Administración Pública sirve con objetividad los intereses generales y actúa de acuerdo con los principios de eficacia, jerarquía, descentralización, desconcentración y coordinación, con sometimiento pleno a la Ley y al Derecho.

16 El artículo 55 EBEP establece los principios rectores para el acceso al empleo público, sin embargo no establece disposiciones específicas o criterios aplicables para una extinción mediante despido. 
De este modo, tradicionalmente se entendía que existen diferencias claras entre los empleadores públicos o privados, en relación con su margen de actuación para establecer, y aplicar, los criterios de selección en las extinciones colectivas. En este sentido, los tribunales clasificaban el nivel de libertad que afectaba a dichos criterios conforme a tres grados diferentes de exigencia, dotando a la empresa privada de mayor libertad frente a lo dispuesto para la Administración pública ${ }^{17}$. El primer grado, de menor intensidad, en supuestos de extinciones en empresas privadas por causas económicas donde los criterios, no discriminatorios en ningún caso, pueden enunciarse con carácter genérico siempre que exista relación de causalidad entre el despido y la causa. El segundo grado, de intensidad media, se concreta en despidos por causas organizativas, productivas, tecnológicas o económicas, relacionados con un sector específico de la empresa, siendo ésta de carácter privado. En efecto, serán más precisos que los anteriores, para establecer la relación de causalidad entre la causa del despido colectivo y el concreto trabajador afectado, también ajeno a discriminaciones por motivos ilícitos. Y por último, el grado de intensidad máxima que aparece en despidos colectivos que afectan al sector público administrativo, incluidos dentro del ámbito de aplicación del art. 23.2 CE ajenos a discriminaciones (Roqueta, 2015: 85). En este último caso, los criterios habrán de ser suficientemente específicos para determinar, mediante su aplicación, quienes son los concretos afectados y valorando circunstancias relacionadas con el mérito y capacidad de los trabajadores, especialmente cuando dentro de este colectivo se encuentren trabajadores fijos que hayan superado procedimientos de ingreso en las Administraciones públicas conforme a los principios de igualdad, mérito y capacidad. No obstante, los despidos realizados en el sector público empresarial o fundacional no compartirán, como expondré, la máxima intensidad en la concreción de sus criterios justificativos conforme a esta clasificación.

\section{Dos puntos críticos respecto a los despidos colectivos en el Sector Público: la prioridad de permanencia y los criterios de selección}

Como he señalado repetidamente, la normativa no ha expresado criterio alguno para la preferencia en la designación de las personas afectadas, ni en el sector público ni en el privado, salvo los que pudiesen pactarse en la negociación colectiva o en el acuerdo concluido durante el período de consultas. Sin embargo, tanto el Estatuto de los Trabajadores, como el RDDC, sí que garantizan la prioridad de permanencia a los representantes de los trabajadores frente a de-

17 STSJ de Granada de 29 de noviembre de 2012 (proc. 8/2012) y Murcia de 9 de julio de 2012 (proc. 8/2012), reproducidas también en la SAN 59/2014, de 28 de marzo (proc. 499/2013). 
cisiones extintivas, e introducen -o sugieren - otras nuevas prioridades ante los despidos colectivos en las Administraciones públicas, como la que se manifiesta en relación con los trabajadores fijos o la que se pueda negociar con motivo de la negociación colectiva o durante el procedimiento de extinción. No obstante, aunque se consideraba que tanto la prioridad de permanencia, como el establecimiento de criterios de selección conforme a los criterios de igualdad, mérito y capacidad, se aplicaban al Sector público en su conjunto, la jurisprudencia y la doctrina han diferenciado claramente dos regímenes de actuación. Por ello, abordaré en el presente epígrafe ambas cuestiones.

\subsection{La prioridad de permanencia en despidos colectivos}

En lo que respecta a la prioridad de permanencia es sabido que, en primer lugar, el RDDC incluye, en su artículo 13, una mención específica acerca de la permanencia de los representantes de los trabajadores - estatutarios y sindicales - y los delegados de prevención, siguiendo lo previsto en los artículos 51.5 y 68.b) del Estatuto de los Trabajadores, el artículo 10.3 de la Ley Orgánica de Libertad Sindical, así como, el artículo 37.1 de la Ley de Prevención de Riesgos Laborales, que también son de aplicación al sector público. El precepto del RDDC se limita a reproducir las reglas previstas en otras normas legales, que condicionan la libertad de elección del empleador, tanto público como privado, en el sentido indicado.

En segundo lugar, a esta previsión ha de incorporarse una particularidad específica para el sector público, aunque presenta una redacción confusa. Se establece la prioridad de permanencia para el personal laboral fijo que hubiese adquirido esta condición a través de un procedimiento selectivo de ingreso — de acuerdo con los principios de igualdad, mérito y capacidad-cuando así lo establezcan dichos entes, organismos y entidades ${ }^{18}$. En cualquier caso, es visible que la protección que otorga la prioridad de permanencia en ambos casos no supone, como es lógico, un blindaje frente a cualquier extinción contractual, de hecho los que cuentan con prioridad de permanencia también pueden verse afectados por el despido cuando éste afecte a todo el personal (Rodríguez-Pińero, 2013: 246-251), sin perjuicio de que haya de justificarse en el expediente esta inclusión.

Esta segunda prioridad, tal como figura redactada en el Estatuto de los Trabajadores, parece que se conforma con carácter potestativo (Rodríguez, 2013:26), es decir, que no opera de forma automática, sino que habremos de estar a la decisión tomada por los entes, organismos y entidades. Sin embargo, a juicio de parte de la doctrina se trata más que de una facultad discrecional

18 Tal como se ha expresado, recogido en la Disposición Adicional 16. a del Estatuto de los Trabajadores vigente. 
de una habilitación a su favor si se constata que no se ha realizado (Roqueta, 2013: 154-155; Rodríguez-Piñero, 2013: 242-243), opinión que comparto. A tal efecto, en el supuesto de que no se haya materializado dicha previsión, la Secretaría de Estado de las Administraciones Públicas o autoridad equivalente en el ámbito de las Comunidades Autónomas (en adelante CCAA), podrá establecer la aplicación de dicha prioridad en los procedimientos que afecten al personal laboral de sus respectivos ámbitos, aun cuando el Departamento, Consejería, organismo o entidad que promueva el procedimiento no lo hubiera previsto anteriormente, si, a la vista del expediente, entendiese que es aplicable. Es decir, cuando se entienda que es adecuada a las circunstancias concurrentes, teniendo en cuenta la ordenación general en materia de recursos humanos y el interés público $^{19}$. Ello requiere que a posteriori, por los motivos indicados, pueda tomarse una decisión que garantice la aplicación de dicha prioridad puesto que en supuestos de no inclusión podría ocasionar que todo el personal laboral estuviese afecto a las medidas extintivas, sin distinciones o prioridades entre ellos.

Igualmente, el RDDC prevé que el Ministerio de Hacienda y Administraciones Públicas o autoridad equivalente en el ámbito de las CCAA, cuando se trate, respectivamente, de procedimientos que afecten al personal laboral de la Administración General del Estado o a las CCAA, podrá también acordar la aplicación de la prioridad, que tendrá carácter vinculante en los términos que figuren en el acuerdo o resolución que se dicte y que habrá de remitir al Departamento, Consejería, organismo o entidad que hubiese iniciado el procedimiento, así como, a la representación de los trabajadores y a la autoridad laboral ${ }^{20}$. Sin embargo, sobre este particular, considero que no estamos ante una facultad discrecional, sino un auténtico derecho del personal laboral fijo, habida cuenta la prioridad establecida en la Ley, que ha sido confirmada por la jurisprudencia ${ }^{21}$ y la doctrina científica (Roqueta, 2015: 83; Purcalla, 2015: 22).

Hay que de puntualizar dos cuestiones sobre la aplicación de esta prioridad. En primer lugar, la norma legal y la reglamentaria han establecido como criterio de prevalencia el carácter fijo de los trabajadores, frente a los temporales o indefinidos no fijos ${ }^{22}$, marcando una diferencia que se basa en la realización de proce-

19 Artículo 41.2 RD 1483/2012.

20 Artículo 43 RD 1483/2012.

21 Entre otras, sentencias del Tribunal Superior de Justicia de Andalucía, de 20 de marzo de 2013 (recurso 11/2012) y de Cataluña, de 17 de junio de 2013 (recurso de Suplicación núm. $1971 / 2013)$

22 Alguna sentencia considera que no es necesario el respeto de la prioridad de permanencia para el trabajador fijo, pudiendo extinguirse dichas relaciones con anterioridad a la de los temporales STSJ de Madrid (sección cuarta) de 9 de abril de 2013 (Recurso de Suplicación núm. 18/2013) donde se abordó esta prioridad en el Ente Público Telemadrid. Este criterio fue confirmado por el Tribunal Supremo (Sala de lo Social) en sentencia de 26 marzo 2014 (Recurso de Casación núm. 158/2013). Puede consultarse la distinción sobre el personal al servicio de las Administraciones Públicas en (Lalaguna, 2012: 7-9). 
dimientos selectivos al acceso al empleo público, aunque sin distinguir el tipo de acceso que hubiese realizado el trabajador: oposición, el concurso-oposición y el concurso de méritos ${ }^{23}$, dotándolos de igual valor, e igualando la desigualdad (Roqueta, 2013: 151-152). En segundo lugar, la prioridad tampoco establece otros criterios adicionales que pudiesen ayudar a interpretar su sentido, puesto que no diferencia si opera frente a cualquier extinción, o tiene que matizarse su ámbito de aplicación, es decir, "si la garantía se mantiene dentro del ámbito de afectación de la causa extintiva o si, por el contrario, puede superar ese ámbito, y extenderse a otros ámbitos que quedan fuera de esa afectación, siempre que en ese ámbito existan puestos de trabajo funcionalmente equivalentes a los afectados y, por tanto, intercambiables" (Roqueta, 2013: 153-154). Esta última cuestión será decisiva para concretar los despidos y la práctica negociadora en el período de consultas, pero también ha generado diversas interpretaciones, tanto en un sentido como en otro.

Ahora bien, es preciso referir que igualmente se planteó la duda de si esta prioridad de permanencia afectaba al conjunto del sector público o exclusivamente a las Administraciones públicas. A tal efecto, diferentes voces doctrinales hacían referencia a un criterio diferenciador para la posible aplicación, como que lo significativo no era la naturaleza de la entidad en cuestión, sino el tipo de vínculo y forma de acceso al puesto conforme a lo previsto en el Estatuto Básico del Empleado Público (Purcalla, 2015: 21-23; Roqueta, 2015: 86), criterio seguido por algunos Tribunales Superiores de Justicia ${ }^{24}$. Sin embargo, esta interpretación no se ha mantenido por el Tribunal Supremo, que en distintas sentencias descarta para todos aquellos que no sean Administraciones públicas - o equiparados conforme a lo previsto en el artículo 3.2 de $\mathrm{RD}$ Legislativo 3/2011- , sin diferenciar conforme a la vinculación o acceso al puesto, la aplicación de dicha prioridad de permanencia ${ }^{25}$. Conforme a lo anteriormente expuesto no habrá de

23 Artículo 61.7 EBEP.

24 Vean Sentencias del Tribunal Superior de Justicia de Castilla y León, como la de 9 de octubre de 2013 (recurso 1277/2013), de 4 de diciembre de 2013 (recurso 1318/2013) y 11 de marzo de 2015 (recurso 2177/2014). En estos casos, aunque algunas han sido recurridas ante el Tribunal Supremo, dichos recursos fueron inadmitidos por falta de contradicción, por ello, no se resolvió sobre este particular. A tal efecto, el TSJ señala en la mencionada sentencia de 11 de marzo, fundamento de derecho séptimo que "(...) De ahi que, aunque aparentemente la disposición adicional 20. a del Estatuto de los Trabajadores la considere solamente un criterio de selección en los despidos colectivos en las Administraciones Públicas, en realidad se trate de una preferencia para el mantenimiento del empleo público cuando se produzcan situaciones de concurrencia como la que aqui nos ocupa, por cuanto responde a un imperativo constitucional. Por tanto, como ocurre, por ejemplo, con la preferencia de los representantes legales o sindicales de los trabajadores, en el caso de las Administraciones Públicas hemos de considerar la misma una preferencia legal general, aplicable en todo tipo de situaciones concurrenciales relativas al acceso o la pérdida del empleo público, que beneficia a los trabajadores fijos sobre los indefinidos no fijos.».

25 Así puede apreciarse que el Tribunal Supremo lo establece en la STS (Sala de lo Social, Sección $1 .^{\text {a) }}$ de 20 octubre 2015 (recurso de casación 172/2014), donde literalmente hace constar, en su Fundamento de Derecho undécimo (2), frente a la impugnación que alega la inaplicación del principio de prioridad de permanencia para el personal laboral fijo «es cierto que en la referida DA Vigésima ET se 
respetarse la prioridad de permanencia del personal laboral fijo que establece el art. 41 del RD 1483/2012, debido a que

... dicho precepto, relativo a la "prioridad de permanencia en el ente, organismo o entidad pública», por su propia ubicación en el Capítulo II de la norma es de aplicación a las Administraciones Públicas, pero no a los entes, organismos y entidades que forman parte del Sector Público, al que pertenece el Ente demandado, cuya regulación se encuentra en el Capítulo I anterior ${ }^{26}$.

Por último, se establece que la prioridad de permanencia podrá extenderse a otros colectivos determinados en virtud de lo previsto en convenio colectivo o en el acuerdo alcanzado durante el período de consultas, tales como trabajadores con cargas familiares, mayores de determinada edad o personas con discapacidad $^{27}$. Esta previsión no constituye una obligación a la hora de negociar convenios colectivos, ni tampoco tendrá que incorporarse necesariamente en el período de consultas, sino que se ofrece como un criterio orientativo que podrá tenerse en cuenta a la vista de las circunstancias extintivas ${ }^{28}$.

\subsection{La introducción de criterios de selección}

La cuestión de la delimitación subjetiva de los afectados requiere, también, de un análisis en este trabajo. Como se ha referido repetidamente, la empresa privada, en ejercicio de la libertad de empresa, puede seleccionar el personal que desee ante la contratación, al igual que ante la extinción. Esta facultad solo se encuentra limitada por las prioridades de permanencia previstas en la normativa, así como, la protección frente a cualquier discriminación.

Por otro lado, en lo que acontece al sector público, hay que matizar que se encuentran sujetos a los principios constitucionales previstos en los artículos 9.3

dispone que "Tendrá prioridad de permanencia el personal laboral fijo que hubiese adquirido esta condición, de acuerdo con los principios de igualdad, mérito y capacidad...». Pero no es menos indiscutible que:

1. $\left.{ }^{\circ}\right)$ Lo subordina a que "asi lo establezcan los entes, organismos y entidades»; y

2. ${ }^{\circ}$ Lo limita a aquellos "a que se refiere el párrafo anterior», que exclusivamente trata de "los entes, organismos y entidades a que se refiere el artículo 3.2 del texto refundido de la Ley de Contratos del Sector Público". Precepto — aprobado por el RD Legislativo 3/2011, de 14/Noviembreque se refiere a los entes que dentro del sector público tienen "la consideración de Administraciones Públicas", y entre los que no están —obviamente-las sociedades mercantiles estatales, como TRAGSA [nos remitimos a lo que precedentemente hemos expuesto acerca de su naturaleza jurídica]». Aunque la sentencia cuenta con dos votos particulares, en ninguno de ellos se discute la referida interpretación.

26 Sentencia del Tribunal Supremo (Sala de lo Social), de 24 marzo 2015 (Recurso de Casación 217/2014).

27 Artículo 13.2 RDDC.

28 Para parte de la doctrina científica, se trata de medidas de soft law. (Rodríguez-Pińero, 2013: 249). A tal efecto, al menos hasta ahora, no ha sido tenido en cuenta en la práctica negocial, quizá motivado por la novedad de la medida. 
y 14 de la CE. Es decir, han de garantizar la interdicción de la arbitrariedad de los poderes públicos y el respeto al principio de igualdad. Así, la Administración, en su actuación, se verá sometida a criterios no discrecionales ni arbitrarios. De esta forma, la doctrina expresa que «la Administración en cuanto actúa como persona jurídica en el comercio está sujeta al principio dispositivo de libertad contractual, pero en cuanto ente público que vela por los intereses sociales ha de ver limitada su actuación» (Lalaguna, 2012: 10).

Esta situación, no obstante, contrasta con aquellas extinciones realizadas en el sector público, que no Administración pública, cuyas pautas de actuación siguen criterios generales. En ocasiones, la compleja estructura interna que presentaban ha permitido que se manifestaran vacilaciones respecto a las disposiciones que se le venían a aplicar. De hecho, la circunstancia expresada ha requerido que los tribunales realicen con carácter previo la calificación de su estructura organizativa (De Soto, 2015: 103). Así, podemos señalar dos supuestos paradigmáticos en lo que respecta a la aplicación de criterios en estas extinciones, en primer lugar, los que se realizan en los distintos grupos audiovisuales de titularidad pública y, en segundo lugar, los atinentes a la empresa Tragsa.

Por un lado, en lo que respecta a los grupos audiovisuales, los despidos colectivos han aplicado pautas para la selección de los afectados de carácter genérico, debido a que dichas sociedades de radiotelevisión no les son de aplicación lo instituido para las Administraciones públicas, en cuanto a la delimitación de las causas y en lo atinente a los criterios de selección al tratarse de sociedades mercantiles de capital público ${ }^{29}$. Por ello, los expedientes de regulación de empleo de la televisión de la Comunidad Valenciana establecieron unos criterios de designación en base a la adscripción del puesto de trabajo que fuera a ser afectado por su eliminación o redimensionamiento, con referencia a la experiencia profesional, a la formación relativa al puesto, a la polivalencia funcional, a las buenas prácticas profesionales, a la situación de excedencia voluntaria y el menor absentismo, al mayor coste de su contrato de trabajo o las aptitudes de gestión de equipo y a las habilidades de interacción social ${ }^{30}$. Estos criterios se concretaron posteriormente mediante una clasificación ordenada, valorando, prioritariamente, la posible incorporación mediante oposición (requisito relacionado con la igualdad, mérito y capacidad). También, la asignación al puesto efectivo de trabajo y/o la titulación específica para el puesto ${ }^{31}$. Igualmente, la prestación efectiva de servicios en los últimos tres años, computando como

${ }^{29}$ A diferencia de los Entes Públicos de radiotelevisión, a los que sí que le son de plena aplicación.

30 STSJ Comunidad Valenciana, (Sala de lo Social, Sección 1.a), Sentencia núm. 2338/2013, de 4 noviembre, Procedimiento núm. 17/2012, hecho probado 6. ${ }^{\circ}$.

31 En esos casos una titulación homologable para el caso de los técnicos que cursaron imagen y sonido, ya que en la actualidad se han convertido en Ciclos Formativos Superiores). 
tiempo de trabajo efectivo determinadas vicisitudes del contrato como los permisos por maternidad, riesgo para el embarazo, excedencia legal por cuidados de hijos y padres, accidente de trabajo o enfermedad profesional y enfermedades graves o crónicas, siempre que sean justificadas por el trabajador. De este mismo modo, también se valora el no haber tenido periodos de excedencia voluntaria o de permisos sin sueldo durante toda la vida laboral del trabajador. Asimismo, se atiende a la capacitación profesional relacionada con el puesto, así como, la polivalencia, cuando el trabajador demuestra que ha sido capaz de ejercer otros puestos de trabajo o, sin haberlo hecho, si lo justifica. Por último, otros aspectos considerados serían la «fidelidad a la empresa», al no haber ejercido simultáneamente otra actividad ajena al Grupo.

Ahora bien, de nuevo hay que referir que dichas extinciones se realizaron conforme a las valoraciones efectuadas por una comisión técnica de valoración, y una subcomisión, tras la entrega por los trabajadores de un formulario, cuyo resultado no se da a conocer salvo por la designación concreta del trabajador afectado. Así, la selección, además de incorporar cuestiones de carácter estrictamente personal, no parece que se hiciese conforme a derecho, lo que se evidencia puesto que se realizaron varias extinciones previamente a la valoración técnica funcional final que se realiza por el Ente público ${ }^{32}$. Sin embargo, en otras televisiones regionales se ha utilizado para la selección de los trabajadores afectados criterios generales como la antigüedad o la polivalencia, justificándose también la permanencia de determinadas personas, por la versatilidad del trabaja$\operatorname{dor}^{33}$.

Por otro lado, en segundo lugar, hay que reseñar el asunto referente a la sociedad mercantil estatal Tragsa, resuelto en primera instancia por la Audiencia Nacional declarando su nulidad, precisamente por la imprecisión de los criterios de selección. Sin embargo esta sentencia fue revocada en casación por el Tribunal

32 Sin embargo, a juicio de la sala lo más importante a la hora de declarar la decisión nula fue el hecho de haber modificado a lo largo de todo el procedimiento las causas que justificaron las extinciones y que diferían entre el ente público y las sociedades mercantiles de capital público afectas a él, como es el caso de una televisión. Aquí, el hecho de haber modificado dichos criterios establece una diferencia entre los trabajadores inicial o posteriormente afectados que sin duda quiebran el principio de igualdad que tiene que tener la administración en su actuación, STSJ de Madrid (sección cuarta) de 9 de abril de 2013 (Recurso de Suplicación núm. 18/2013) donde se abordó esta prioridad en el Ente Público Telemadrid. Este criterio fue confirmado por el Tribunal Supremo (Sala de lo Social) en sentencia de 26 marzo 2014 (Recurso de Casación núm. 158/2013).

33 STSJ Murcia (Sala de lo Social, Sección 1.a), de 14 de octubre de 2013, expuso estos criterios para el despido colectivo en la televisión murciana, lo que a juicio de la STS (Sala de lo Social), de 24 marzo 2015, Recurso de Casación núm. 217/2014, se consideró que habida cuenta de que se trata de sector público y no Administraciones públicas, "Teniendo en cuenta que la empresa adujo criterios de selección, "aunque fuera de forma genérica (antigüedad, polivalencia)" como refiere la sentencia recurrida; ello evidencia la inexistencia de la causa de nulidad con base a la irregularidad de insuficiencia de criterios en la designación de los trabajadores afectados", por ello parece permitir una cierta inconcreción siempre que, al menos, indique las líneas en las que se basa la extinción. 
Supremo ${ }^{34}$, lo que ha sido objeto de crítica por parte de la doctrina ${ }^{35}$. En este supuesto concreto, en lo que respecta a los criterios de selección, se elaboró un manual por la empresa, que no fue objeto de negociación. El referido manual incluía además de criterios valorables como la formación o la experiencia, incluye otros como el absentismo y la actitud, ésta última atendiendo a los siguientes factores: identificación y compromiso con la empresa, implicación en la consecución de los objetivos, cumplimiento de horarios, normas y procedimientos establecidos trabajo en equipo y polivalencia (capacidad para adaptarse a cambios y otras funciones). Todo ello será evaluado por los responsables de la empresa a través de un sistema informático que integra sus puntuaciones, coste de indemnización por despido y obligación de hacer una aportación al Tesoro Público.

La sentencia de la Audiencia Nacional había cuestionado los mencionados criterios por diversas razones. Por un lado, porque la experiencia y la formación son analizadas por los responsables de la empresa de forma subjetiva, por otro lado, porque «el factor de actitud podia ser apreciado negativamente conforme a los siguientes parámetros, mostrarse distante o esquivo hacia temas relacionados con la organización, actitud critica con cualquier aspecto de la empresa, sin aportar elementos constructivos de cambio y mejora en sus comentarios y actuaciones, percibir cualquier alteración en la dinámica laboral como un problema, mostrarse reacio a cambiar de postura u opinión, demostrando poca capacidad de escucha o poca disponibilidad a asumir elementos nuevos, como cambios en el procedimiento, normativas o tareas ${ }^{36}$. A mayor abundamiento, el personal encargado de valorar introducía los datos en un programa informático sin dar audiencia al interesado. A la vista de estos hechos, la Audiencia estimó la nulidad del despido colectivo ya que entendía que la Administración pública, y también los entes públicos incluidos en el ámbito de aplicación del artículo 23.2 de la CE, tenían que ser suficientemente específicos en los criterios de selección, a partir de magnitudes mensura-

34 SAN 59/2014, de 28 de marzo (proc. 499/2013) y STS (Sala de lo Social, Sección 1.a), de 20 de octubre de 2015, Recurso de Casación núm. 172/2014, se consideró que habida cuenta de que se trata de sector público y no Administraciones públicas, los criterios son adecuados, ya que en primer lugar se extinguirán los puestos de "trabajadores cuyo puesto de trabajo y unidad organizativa o centro de trabajo de adscripción se encuentren comprendidos en el cuadro recogido en el apartado 5.4 de la presente Memoria Explicativa, que se sustenta en el informe técnico de causas organizativas y productivas en el que se describe la metodología y los parámetros técnicos para la determinación de los puestos excedentarios», para, en segundo lugar, los trabajadores "serán seleccionados por la empresa según criterios de evaluación multifactorial, tales como la formación, experiencia en el puesto, polivalencia entendida como la capacidad para asumir cambios y adaptación a otras funciones, trabajo en equipo, grado de implicación en la consecución de objetivos, cumplimiento de horarios, normas y procedimientos establecidos, absentismo, costes. La aplicación de estos criterios será plenamente respetuosa con los derechos fundamentales y legislación vigente», por ello el Tribunal Supremo convalida la decisión extintiva y revocando la sentencia de la Audiencia Nacional.

35 Con un análisis muy pormenorizado sobre la sentencia puede consultarse el Blog del prof. Rojo, www.eduardorojotorrecilla.es.

36 Hecho probado decimotercero de la sentencia de la SAN 59/2014, de 28 de marzo. 
bles para poder determinar mediante su aplicación quienes son los trabajadores afectados, criterios que estarán fundados en circunstancias dirigidas a valorar el mérito y capacidad de los trabajadores. Igualmente, considera que estos criterios vulnerarían lo dispuesto en los artículos 9.3 y 23.2 de la CE, que proscriben la arbitrariedad en el acceso al empleo público lo que, a juicio de la Audiencia, debía entenderse también a la pérdida de la condición del empleado público, ya que de otra forma se vaciaría de contenido al derecho. Como se ha anticipado, el Tribunal Supremo ha desvirtuado esta interpretación, lo que considero es un cambio desfavorable, con respecto al criterio de la Audiencia. Por tanto, bastará con alegar un criterio no discriminatorio, mínimamente justificado, para acreditar el cumplimiento de la norma.

Conforme a lo anterior, es visible que a los despidos que se producen en el sector público empresarial o fundacional, se les aplica el criterio de libre elección de los trabajadores. Por ello, el nivel de libertad que afecta a estas extinciones será, dependiendo del supuesto en el que se materialicen, de intensidad menor o media, tal como se expuso en páginas anteriores, sin que en estos casos compartan con la Administración pública el grado de intensidad máxima. Este hecho, a mi juicio, supone una importante debilitación de nuestro Estado de derecho. En consecuencia, se desvirtúa la naturaleza de estas entidades permitiéndoles, respecto a la selección de los trabajadores afectados, que sean ajenas a sus principios de actuación y dotándolas, para estos aspectos, de un carácter enteramente privado, tan solo requerirá de una mera alegación de la justificación, lo que a mi entender es merecedor de censura jurídica.

$\mathrm{Al}$ mismo tiempo, en lo que respecta a la posibilidad de extinciones contractuales por la Administración Pública deberíamos encontrarnos ante un distinto escenario. Sin embargo, en la práctica, como se verá, su actuación no difiere en exceso de lo previsto para las entidades privadas. El hecho es que la norma lo único que viene a referir es la necesidad de ceñirse a criterios objetivos, que deben ser adecuadamente precisados. Aquí, por tanto, es preciso que la Administración Pública limite su discrecionalidad al seleccionar al personal afectado, vinculando los puestos amortizados con los empleados públicos que se van a despedir (Cruz, 2012: 39).

Por ello, ante la ausencia de criterios generales, la doctrina ha intentado paliar esta ausencia introduciendo unos criterios ordenadores de carácter orientativo frente a la decisión de extinguir. Así, se considera que se verán afectados en primer lugar, los trabajadores con contrato temporal, en segundo lugar los trabajadores indefinidos no fijos de plantilla y demás empleados cuyo contrato esté aquejado de irregularidades, en tercer lugar, los trabajadores que ocupen puestos de trabajo a suprimir, en cuarto lugar, los resultados obtenidos por los trabajadores en la evaluación del desempeño y, en su defecto, la puntuación alcanzada en el concurso de provisión de puestos de trabajo o de acceso y por último 
la antigüedad (Roqueta, 2012: 151) ${ }^{37}$;. Otros sectores de la doctrina, también hacen una propuesta de otros mecanismos, como «los concursos de méritos negativos", es decir, atendiendo a una comparación basada en la formación, conocimientos e historial, y seleccionando a los de peor calificación para su extinción (Rodríguez-Pińero, 2013: 251). En cualquier caso, estos criterios tienen que recoger la prioridad de los trabajadores fijos establecidos para la Administración, y también habrán de respetar necesariamente las demás legalmente previstas en favor de los representantes legales o preventivos.

A mi juicio, a día de hoy los criterios que se articulan son manifiestamente insuficientes, puesto que no se ha implantado en la Administración sistema alguno de evaluación del desempeño. Por ello, atender a la puntuación que obtuvo el trabajador en su acceso al empleo, evita tener en cuenta el desarrollo profesional que hubiese podido realizar durante su carrera profesional, y otro criterio podría resultar arbitrario e injusto. Por tanto, en mi opinión, deben tenerse en consideración dos variables a la hora de extinguir el contrato: primera, la adecuación al puesto del trabajador, es decir, su capacidad concreta y, segunda, los méritos del mismo, pero no solo los iniciales, sino los obtenidos durante su trayectoria, de este modo se valoraría el mérito del trabajador y su capacidad con respecto a las circunstancias concretas del puesto que se va a mantener.

En consecuencia, resulta necesario, por su importancia, establecer un sistema que garantice que las Administraciones Públicas aplican criterios objetivos, ajenos a toda arbitrariedad, ante la necesidad de seleccionar a los trabajadores afectados por las decisiones extintivas (Cruz, 2010: 49). Estos criterios habrán de respetar, en su caso, las prioridades de permanencias legales o pactadas que afecten al sector concreto.

\section{Los criterios de selección y la prioridad de permanencia, un análisis de las decisiones judiciales frente a las extinciones en las Administraciones públicas}

Ante las cuestiones analizadas se nos plantea el siguiente interrogante: ¿̇se están estableciendo criterios objetivos en las extinciones realizadas en la Administración pública? La respuesta no es sencilla puesto que las extinciones resueltas por los Tribunales no siempre analizan cuestiones acerca de la concurrencia formal de criterios objetivos en la extinción.

La aplicación de criterios para la selección —o exclusión en virtud de la prioridad de permanencia - han sido tratados bien porque se entiende que vul-

37 En el mismo sentido, Rodríguez (2013: 25-26). 
neran el principio de igualdad, o bien, con motivo de su posible inclusión en causas discriminatorias, dejando a los Juzgados de lo Social la valoración individual del ajuste a los criterios para la selección, por razones procedimentales. La Ley 36/2011, de 10 de octubre, reguladora de la jurisdicción social (BOE 11 de octubre), en su artículo 124.13, establece que la posible vulneración de criterios objetivos para decidir quiénes son en concreto aquellos trabajadores afectados por el expediente de regulación de empleo no podrán ser objeto de un procedimiento de despido colectivo, ya que la demanda solo podrá fundarse en la falta de inclusión en el expediente de la relación de trabajadores afectados o del criterio utilizado para su elección, o bien, cuando se alegue que dicha decisión se tomó con fraude, dolo, coacción o abuso de derecho o vulnerando derechos fundamentales o libertades públicas. Por tanto, aunque la decisión de extinción tomada sin respetar las prioridades de permanencia establecidas en la ley, convenios colectivos, o acuerdos durante el período de consultas será nula, su revisión habrá de seguir un procedimiento individual y no se resolverá dentro del proceso sobre despido colectivo. Aquí se manifiesta otro problema adicional que ya ha sido puesto de manifiesto por un cierto sector de la doctrina, que considera que la inconcreción de los criterios no puede subsanarse mediante la remisión a los procesos individuales de despido ${ }^{38}$.

De este modo, en general se exponen en diversas resoluciones las razones que deben inspirar la conducta de la Administración a la hora de contratar o extinguir, estableciendo que en la contratación habrá de aplicar sistemas objetivos que permitan la elección de los más idóneos para desarrollar las funciones asignadas a sus puestos de trabajo de acuerdo con los principios constitucionales de igualdad, mérito y capacidad por lo que, consecuentemente, al seleccionar a quienes va a extinguir sus contratos, debe hacerlo pero a la inversa, evaluando su desempeńo, su conducta profesional, su rendimiento y el logro de resultados mediante sistemas de evaluación que se deberían adecuar, en todo caso, a criterios de transparencia, objetividad e imparcialidad ${ }^{39}$.

Igualmente, las sentencias estudiadas abordan las prioridades de permanencia, siempre con respeto de las previstas para los representantes de los trabajadores y de prevención, y exponiendo, respecto a ello, la diferencia de tratamiento entre personal funcionario y laboral — como es sabido los primeros no están

38 Voto particular STS (Sala de lo Social, Sección 1.a) de 25 de junio de 2014, (Recurso de Casación núm. 198/2013) con alegación expresa de las obligaciones derivadas de los compromisos internacionales que se manifiestan en el Convenio n. ${ }^{\circ} 158$, sobre la terminación de la relación de trabajo, 1982, ratificado por Espańa en 1985 y la Directiva 98/59/CE del Consejo de 20 de julio de 1998 relativa a la aproximación de las legislaciones de los Estados miembros que se refieren a los despidos colectivos (DO L 225 de 12.08.1998).

39 ST Juzgado de lo Social núm. 12 de Barcelona de 13 de diciembre de 2011 (Procedimiento núm. 470/2011), anterior a la reforma laboral, referido a la entidad de Derecho público, Instituto Catalán del Suelo. 
afectos a procedimientos de despido colectivo— ${ }^{40}$, y la que se establece entre personal fijo y el personal indefinido no fijo o el temporal ${ }^{41}$.

Ahora bien, en la práctica judicial se manifiestan diversos criterios adoptados en el período de consultas. Algunas extinciones siguen las pautas sugeridas por la norma: como es la edad, las cargas familiares o la discapacidad favoreciendo la permanencia de los colectivos afectados por estas condiciones. De este modo, parece consagrarse con la aplicación de los referidos criterios, una disminución del coste social de los despidos colectivos, cuestión que parte de la doctrina considera muy adecuada (Ginès, 2010: 3-4), ya que estas actuaciones permiten reducir, además del mencionado coste social, los costes individuales de la extinción y, consecuentemente, conllevan efectos sobre el consumo, la inversión, el ahorro, la oferta y la demanda. En esta línea, distintas resoluciones reflejan dichos criterios, fijando un rango de edad concreto, el hecho de que el trabajador sea familia monoparental con hijo a cargo, con pareja en desempleo, cuyo único ingreso dependa de la entidad pública que realiza el expediente o que tenga hijos aquejados de discapacidad ${ }^{42}$.

Ahora bien, el nudo gordiano se encuentra en aquellas — escasas- sentencias que han acometido la revisión de los criterios de selección ${ }^{43}$. Se trata, generalmente, de extinciones en el ámbito de la Administración local o Diputaciones, donde algunos expedientes atienden a criterios de edad, así como, al resultado de procedimientos de evaluación continua. En estos casos, se contrasta la existencia de una consulta previa al personal técnico responsable, para preser-

40 Si bien el art. 85.2 del EBEP prevé, por su parte, la posibilidad de que en el futuro se regulen «otras situaciones administrativas de los funcionarios de carrera, en los supuestos, en las condiciones y con los efectos que en las mismas se determinen, cuando (...) por razones organizativas, de reestructuración interna o exceso de personal, resulte una imposibilidad transitoria de asignar un puesto de trabajo o la conveniencia de incentivar la cesación en el servicio activo» lo que hace pensar que no está tan lejana la posibilidad de un despido colectivo para el personal funcionario.

41 STSJ Galicia (sala de lo Social, sección 1.a) de 2 de mayo de 2013 (recurso de Suplicación núm. 10/2013), para la extinción colectiva de la Diputación de Orense, que se vio confirmada por la del Tribunal Supremo (Sala de lo Social, Sección 1.a) de 23 mayo 2014 Sentencia núm. 396/2016 de 6 mayo, (recurso de Casación núm. 179/2013); en el mismo sentido, STSJ de Madrid, (Sala de lo Social, Sección 1.a), en el Recurso de Suplicación núm. 54/2016. Igualmente, se refieren en distintas sentencias la diferencia frente a la extinción del contrato de un trabajador indefinido no fijo y otro que ocupa una plaza de interinidad por vacante STSJ Madrid (sala de lo social, sección 4. ${ }^{\circ}$ ) de 14 de junio de 2013 (Recurso de Suplicación núm. 1398/2013) y STSJ Islas Baleares de 9 de abril de 2013 (Recurso de Suplicación núm. 272/2012).

42 STSJ Comunidad Valenciana de 12 de marzo de 2013 (proceso 5/2013), que fue convalidado por el Tribunal Supremo en su sentencia de 21 de mayo de 2014, (recurso de casación número $162 / 2013)$.

43 Son numerosas las que refieren, pero no abordan, los criterios, como la STSJ de las Islas Canarias, Las Palmas (Sala de lo Social, Sección 1.a), de 19 de diciembre de 2012 (Recurso de Suplicación núm. 17/2012), referido al Ayuntamiento de Gáldar, que habla de su existencia aunque no detallando cuestión alguna sobre ella, siguiendo la misma línea, la sentencia de casación sobre ella STS (Sala de lo Social, Sección 1.a), de 16 de abril 2014 (Recurso de Casación núm. 57/2013). 
var el empleo de aquellos con mayor competencia técnica, formación, experiencia y polivalencia, respetando la prioridad de permanencia para el personal laboral fijo que hubiese adquirido esta condición conforme a los referidos principios de mérito y capacidad. Igualmente, también se ha tenido en consideración la valoración del desempeño de funciones concretas, como las de naturaleza comercial, y una mayor cualificación profesional, conjuntamente con criterios de mayor antigüedad en el área de trabajo, mayor antigüedad en la empresa, mayor edad, mayor experiencia profesional, mayores cargas familiares y mayor grado de discapacidad ${ }^{44}$, que vienen a unirse a la mencionada prioridad de permanencia del personal laboral fijo. Estos criterios se acomodaban —al menos aparentemente- a los principios exigidos en la actuación de la Administración, ya que constituyen causas objetivas y razonables ajustadas al art. $103 \mathrm{CE}$ respetando, por consiguiente, los principios de mérito y capacidad para el acceso a la función pública. Sin embargo, en la práctica, se vieron afectados por actuaciones arbitrarias.

Efectivamente, a la vista del contenido de las sentencias, se aprecia que en la determinación del personal afectado, y a los efectos de su valoración, no se tuvo en cuenta el uso de información de carácter veraz, sino que se consideraron otros motivos, dotando de una apariencia de imparcialidad a los criterios, pero que no se correspondían con la realidad de la decisión extintiva. Asi, en algunos casos se evaluó al personal en función de su carácter problemático o quejica, e incluso acogiendo como cierta la rumorología imperante en el centro de trabajo $^{45}$. Otras extinciones utilizan como criterio para la elección de los trabajado-

44 STSJ Comunidad Valenciana de 12 de marzo de 2013, sin embargo, este criterio fue impugnado por la representación de los trabajadores por entenderlo arbitrario ya que consideraba que no existía distinción entre trabajos comerciales y los administrativos, puesto que existía en la empresa polivalencia funcional, lo que no fue aceptado por la sala.

45 Sorprende por ello, el contenido de la STSJ Andalucía de 20 de marzo de 2013 (Sevilla), referido a la regulación de empleo del Ayuntamiento de Jerez. Sin embargo, pese a la evidencia de estas decisiones desviadas, en función de las diferentes categorías, ya que se confirmó que el criterio se basaban en "quién trabajaba mejor o peor, era problemático, quejica, en base a la rumorología, la actitud ante el trabajo, si eran vagos y que los informes eran verbales, no valorando la formación, sino quien trabajaba bien, era el día a día y esa documentación se destruyó, porque no eran buenos trabajadores y eran conflictivos, con encarecimiento de los servicios, y hay quien no hace nada y el desconocía el currículo", la sala no consideró como nula la decisión tomada al estimar parcialmente las demandas y reconocer la improcedencia de los despidos. Esta decisión ha sido convalidada por el Tribunal Supremo (Sala de lo Social, Sección1.a), en su sentencia de 25 de junio de 2014 (Recurso de Casación núm. 198/2013), aunque con un voto particular que manifiesta su discrepancia con la decisión mayoritaria de la Sala al entender que aunque formalmente se concretan criterios objetivos, en la práctica se trata de una decisión arbitraria e irrazonable, opinión que comparto absolutamente. Así se expresaba que "en definitiva, los principios de igualdad, mérito y capacidad que son esenciales y exigibles para el acceso a las diversas formas de empleo público no se tuvieron realmente en cuenta para determinar, a la inversa, quienes debian resultar directamente afectados por la extinción contractual colectiva; $y$, además, como concluia la sentencia ahora impugnada, en la aplicación del sistema o criterio de evaluación continua la Administración pública empleadora incurrió en una absoluta arbitrariedad y que, como quedó acreditado 
res afectados, uno tan simple como la falta de inclusión en la relación de puestos de trabajo (RPT), la supresión de servicios no obligatorios - y su correspondiente vinculación al mismo- y criterios objetivos como titulación, antigüedad y edad ${ }^{46}$, pero sin justificarlo adecuadamente. E incluso, se manifiestan extinciones conforme a un criterio que se basa, en primer lugar, en el carácter del personal extinguiendo al de naturaleza temporal e interina y, en segundo lugar, sobre el personal indefinido prioriza la permanencia de aquellos trabajadores considerados «imprescindibles», lo que se acogió como un criterio razonable y suficientemente justificado por la sala ${ }^{47}$. Por tanto, se evidencia el carácter subjetivo de las razones esgrimidas.

Por otra parte, también he de señalar aquellos supuestos, como fue en el caso de las pruebas para orientadores laborales, en los que se incorpora un criterio de valoración basado en el orden de puntuación obtenido en las pruebas de acceso específicas, convocadas para la ejecución del programa, a través del que fueron seleccionados los que forman parte del colectivo sujeto al expediente de regulación de empleo. En el asunto que se resolvió, no obstante, el criterio viene referido al momento de selección de los trabajadores, sin tener en cuenta la situación posterior de méritos o rendimiento en el desempeńo de su $\operatorname{actividad}^{48}$,

en la práctica de la prueba, a juicio subjetivo del técnico o del delegado y, con una valoración personal, subjetiva y arbitraria, carente de la aplicación de cualquier parámetro objetivo, fueron determinados los trabajadores afectados por la decisión extintiva, sin tenerse en cuenta ni su competencia técnica, ni su formación, ni su experiencia, ni la polivalencia».

46 STSJ de las Islas Canarias, Las Palmas (Sala de lo Social, Sección 1. ${ }^{\text {}}$ ), de 19 de diciembre de 2012 (Recurso de Suplicación núm. 21/2012), referido al Ayuntamiento de la Oliva, sin que al declarar su nulidad por falta de causa tratase el ajuste o no a derecho de los criterios para la selección. Esta misma circunstancia se reprodujo en la sentencia de casación STS (Sala de lo Social, Sección 1. ${ }^{\text {a) }}$, de 18 febrero 2014 (Recurso de Casación núm. 59/2013).

47 STSJ Galicia (Sala de lo Social, Sección 1.a) de 2 de mayo de 2013, (Recurso de Suplicación núm. 10/2013), para la extinción colectiva de la Diputación de Orense. A tal efecto, como criterio de justificación, junto a los mencionados en otros apartados, se refiere que "El despido colectivo afecta solamente a personal laboral indefinido (no al personal laboral fijo de la Entidad demandada). $Y$ entre el personal indefinido no afecta a quienes tengan la condición de representante de los trabajadores, tampoco afecta a los trabajadores que tenga reconocido un grado de discapacidad igual o superior al 33\%. Se excluye también al personal laboral indefinido que desempeña puestos de trabajo que resultan «imprescindibles", asi como al personal cuyo despido sería antieconómico por estar próxima su jubilación.» No se comprende cómo puede valorarse como suficientemente justificado el criterio de la «imprescindibilidad». Este criterio se convalidó, asimismo, por el Tribunal Supremo (Sala de lo Social, Sección 1.a), en la sentencia de casación, de 23 mayo 2014 (recurso número núm. 179/2013).

48 STSJ Castilla y León, Valladolid (Sala de lo Social, Sección 1. ${ }^{\text {a) }}$ de 28 de mayo de 2013, (Proceso en primera instancia núm. 9/2013), en relación a las extinciones de contratos de personal laboral contratado para la ejecución del plan extraordinario de medidas de orientación, formación profesional e inserción laboral del Servicio público autonómico de empleo de Castilla y León, donde además se hace mención de las diferencias entre personal fijo e indefinido no fijo, refiriendo la objetividad del criterio de selección y estableciendo "(...) que el doble criterio de dar preferencia a quienes han realizado una prueba específica para realizar el cometido de orientador laboral, respecto a quienes no la han realizado es un criterio objetivo y razonable como lo es el dar preferencia a los resultados obte- 
lo que a todas luces resulta poco ecuánime con la realidad presente de los sujetos que se ven afectados por la extinción.

Ante las circunstancias expresadas se evidencia la necesidad de una modificación normativa, con introducción de mecanismos de valoración objetivos, que permitan que la Administración pública se ciña a criterios ajenos a discrecionalidad. Estos criterios no tendrán por qué ser únicos, sino que pueden adecuarse al lugar en el que se ejecute la medida extintiva, siempre condicionados por la preceptiva concurrencia de los principios de igualdad, mérito y capacidad. Conforme a lo anterior, la introducción de un sistema objetivo de evaluación del desempeño es prioritaria, mecanismo que aparece previsto, pero no llevado a cabo, en el propio Estatuto Básico del Empleado Público. Por tanto, dicha medida simplificaría la aplicación de los criterios de selección, con interdicción de arbitrariedad alguna.

\section{Reflexiones finales}

La adaptación del sector público a instituciones laborales como la del despido colectivo requiere de un ajuste más profundo que el realizado con el RDDC. Es necesario buscar alguna fórmula que garantice que el sector público actúa, en cuestiones como la determinación de las personas afectadas por los despidos, como es dable esperar, es decir, con arreglo a los criterios de no arbitrariedad, supeditando sus acciones al cumplimiento del interés general. Sin embargo, a la vista de lo expuesto en páginas anteriores, es visible que se evidencian dos modelos distintos: por un lado, el que afecta exclusivamente a las Administraciones públicas, con interdicción de arbitrariedad y por ello con menor libertad frente a la determinación de los sujetos afectados. Por otro lado, el del resto de entidades, entes y organismos del sector público, que comparten con la empresa privada los mismos procedimientos y mecanismos, dotados de mayor libertad en su actuación dirigida a la extinción contractual.

nidos en dicha prueba». En este mismo sentido, la STSJ de Cataluña, (Sala de lo Social, Sección 1.a) de 19 diciembre, aunque haciendo referencia a entidades de derecho público que realizan actividades mercantiles y, por tanto, formando parte del Sector público, pero sin constituir Administración pública conforme al artículo 3.1 del RD Legislativo 3/2011, refieren el establecimiento de un sistema de valoración conforme a los criterios establecidos en un manual de competencias, mapa de conocimientos y estudio de cargas de trabajos que, sin embargo en la práctica, no permitió a los trabajadores conocer los resultados globales de las evaluaciones efectuadas. En el supuesto que referimos, cada trabajador afectado recibió con posterioridad al período de tramitación de consultas su valoración personal, lo que parece sembrar una duda razonable sobre la ecuanimidad de las valoraciones. No obstante, a juicio del TS (Sala de lo Social, Sección 1. ${ }^{\text {a) }}$, en sentencia de 18 noviembre 2014, que desestimó el recurso de Casación (núm. 160/2013) interpuesto sobre la anterior, quizá porque no se sustentó adecuadamente el recurso. 
La Administración pública, al tener que identificar el número y personas afectadas en los despidos colectivos, ha de justificar y aportar los criterios de selección de los designados. Esta actuación puede suscitar controversias debido a que, se fijan criterios, pero no se concretan unas bases o baremos respecto a su adecuación al puesto, que pudieran permitir una evaluación no arbitraria del desempeño de los sujetos afectados. Así, la normativa únicamente prevé el sometimiento de la Administración al principio de prioridad de permanencia de trabajadores fijos sobre los temporales y, en lo que respecta a la articulación de criterios de selección, no hay reglas inequívocas, sino que dichos criterios necesariamente habrán de ser objetivos, "condicionados por los principios de acceso, promoción y permanencia en los puestos conforme a los criterios de mérito y capacidad» (Cruz, 2012: 39).

En esta línea, por el momento no existen mecanismos que puedan ser utilizados, a diferencia de lo que sucede en grandes empresas, para evaluar el desempeño del empleado público. No obstante lo anterior, existe una previsión, que figura en el artículo 20 del Estatuto Básico del Empleado Público, sobre el establecimiento de sistemas de evaluación del desempeño, tanto de los funcionarios como del personal laboral, que aún no se ha puesto en práctica. El establecimiento de estos sistemas, que deben adecuarse a criterios de transparencia, objetividad, imparcialidad y no discriminación, permitiría valorar la conducta profesional y el rendimiento o el logro de resultados del trabajador y, si bien no están previstos para ser utilizados frente a las extinciones, podrían servir de orientación. No obstante, sería conveniente replantearse la introducción de los sistemas de evaluación, lo que hasta ahora no se ha acometido, posiblemente por motivos económicos, y la posible vinculación de los mismos a los despidos. Ello requeriría, en todo caso, de una modificación normativa sobre el particular.

$\mathrm{Al}$ mismo tiempo, las normas que fijen los criterios y mecanismos generales en materia de evaluación del desempeño pueden formar parte de la negociación colectiva en virtud de lo establecido en el artículo 37.1.d) del Estatuto Básico del Empleado Público. Por tanto, a mi juicio, es preciso que los sujetos negociadores tomen conciencia de esta necesidad y desarrollen en los convenios colectivos esta cuestión incorporando un modelo flexible para todos los empleados públicos, que pueda atender a sus particularidades, criterios objetivos que permitan mediante la aplicación de baremos destinados valorar el desempeño del empleado, junto a la formación adquirida durante su trayectoria profesional.

En conclusión, todos estos criterios podrían utilizarse como base dentro de los trámites del despido colectivo limitando la discrecionalidad de la Administración que se evidencia en las resoluciones judiciales revisadas. Con ello se simplificaría la evaluación de los sujetos afectados, conforme a baremos igualitarios, al mismo tiempo que garantizaría una mayor imparcialidad en las decisiones extintivas, respetando el principio de legalidad. 


\section{Bibliografía}

Cantero Martínez, Josefa (2012), «Las medidas de racionalización de plantillas en el empleo público local y en un contexto de contención fiscal», Cuadernos de Derecho Local (QDL), núm. 28, págs. 7-39.

Cruz Villalón, Jesús (2010), «Los expedientes de regulación de empleo en las Administraciones Públicas», Revista de Derecho Social, núm. 49, págs. 17-40.

De Sande Pérez-Bedmar, María (2006), Empleo y prestación de servicios en la Administración Pública, Ed. Lex Nova, Valladolid.

De Soto Rioja, Sebastián (2015), «Los despidos colectivos en el ámbito del sector público. Balance de aplicación tras «casi» tres años desde su reforma», en Reforma de las Administraciones Públicas y Empleo Público, (Gómez Muñoz, J.M., coordinador), Monografías de Temas Laborales, Sevilla, págs. 85-151.

García Muñoz, Manuel (2013), «Despido colectivo por causas económicas en la Administración local». Revista Doctrinal Aranzadi Social volumen 6 núm. 5. Versión digital, documento Westlaw. BIB 2013\1678.

Ginès y FÁbrellas, Anna (2010), «Criterios de selección de los trabajadores afectados por un despido colectivo", Aranzadi Social, Revista doctrinal, núm. 14. Versión digital, documento Westlaw, BIB 2010\2374, págs. 1-11.

Lalaguna Holzwarth, Enrique (2012): «Despidos por causas económicas, técnicas, organizativas o de producción del personal laboral al servicio de las Administraciones Públicas en la Ley 3/2012», Aranzadi Social, Revista doctrinal, núm. 6. Versión digital, documento Westlaw, BIB 2012\2918, págs. 1-22.

López Cumbre, Lourdes (2013), «La contratación laboral en el sector público y los derechos de los empleados públicos: los efectos de la reforma laboral en el empleo público», Revista General de Derecho Del Trabajo y de la Seguridad Social, Iustel, núm. 34.

Purcalla Bonilla, Miguel Angel (2015), «El despido colectivo en la Administración Pública y en sector público», Revista Jurídica de Castilla y León, núm. 37, págs. 1-30.

Rodríguez Escanciano, Susana (2013), «Los despidos colectivos en la Administración pública», ponencia presentada en las XXIV Jornadas Catalanas de Derecho Social (21 y 22 de febrero de 2013), http://www.iuslabor.org/jornades-i-seminaris/ponencies/.

Rodríguez-PiÑERo Royo, Miguel (2013), «El despido colectivo en el sector público», en Estudios en torno a la Reforma Laboral de 2012, (Castellano Burguillo, E. y Feria Basilio, I., dir.), Editorial Punto Rojo, Sevilla.

Rojo Torrecilla, Eduardo, El blog de Eduardo Rojo, www.eduardorojotorrecilla.es

Roqueta Buj, Remedios (2013), La reestructuración de las plantillas laborales en las Administraciones Públicas. La reforma laboral de 2012, Editorial Tirant lo Blanch, Valencia.

Roqueta Buj, Remedios (2015), La selección de los trabajadores afectados por los despidos colectivos, Editorial Tirant lo Blanch, Valencia. 